\title{
The outburst of an embedded low-mass YSO in L1641
}

\author{
A. Caratti o Garatti ${ }^{1}$, R. Garcia Lopez ${ }^{1}$, A. Scholz ${ }^{1}$, T. Giannini ${ }^{2}$, J. Eislöffel ${ }^{3}$, B. Nisini ${ }^{2}$, F. Massi $^{4}$, \\ S. Antoniucci ${ }^{2}$, and T. P. Ray ${ }^{1}$
}

\footnotetext{
1 Dublin Institute for Advanced Studies, 31 Fitzwilliam Place, Dublin 2, Ireland e-mail: alessio@cp.dias.ie

2 INAF - Osservatorio Astronomico di Roma, via Frascati 33, 00040 Monte Porzio, Italy

3 Thüringer Landessternwarte Tautenburg, Sternwarte 5, 07778 Tautenburg, Germany

4 INAF - Osservatorio Astrofisico di Arcetri, Largo E. Fermi 5, 50125 Firenze, Italy
}

Received 15 November 2010 / Accepted 30 November 2010

\section{ABSTRACT}

\begin{abstract}
Context. Strong outbursts in very young and embedded protostars are rare and not yet fully understood. They are believed to originate from an increase in the mass accretion rate $\left(\dot{M}_{\text {acc }}\right)$ onto the source.

Aims. We report the discovery of a strong outburst in a low-mass embedded young stellar object (YSO), namely 2MASS-J054248480816347 or [CTF93]216-2, as well as its photometric and spectroscopic follow-up.

Methods. Using near- to mid-IR photometry and NIR low-resolution spectroscopy, we monitor the outburst, deriving its magnitude, duration, as well as the enhanced accretion luminosity and mass accretion rate.

Results. [CTF93]216-2 increased in brightness by 4.6, 4.0, 3.8, and $1.9 \mathrm{mag}$ in the $J, H, K_{\mathrm{s}}$ bands and at $24 \mu \mathrm{m}$, respectively, corresponding to an $L_{\mathrm{bol}}$ increase of $\sim 20 L_{\odot}$. Its early spectrum, probably taken soon after the outburst, displays a steep almost featureless continuum, with strong $\mathrm{CO}$ band heads and $\mathrm{H}_{2} \mathrm{O}$ broad-band absorption features, and $\mathrm{Br} \gamma$ line in emission. A later spectrum reveals more absorption features, allowing us to estimate $T_{\text {eff }} \sim 3200 \mathrm{~K}, M_{*} \sim 0.25 M_{\odot}$, and $\dot{M}_{\mathrm{acc}} \sim 1.2 \times 10^{-6} M_{\odot} \mathrm{yr}^{-1}$. This makes it one of the lowest mass YSOs with a strong outburst so far discovered.
\end{abstract}

Key words. accretion, accretion disks - stars: formation - ISM: jets and outflows - infrared: ISM

\section{Introduction}

Most of the stellar mass in low-mass YSOs is assembled within the first $10^{5} \mathrm{yr}$ of their evolution (i.e. class $0,10^{4} \mathrm{yr}$, and Class I, $10^{5}$ yr: see e.g., Lada \& Wilking 1984; Andre et al. 1993). During this stage, the YSO luminosity is thus expected to be dominated by accretion. However, several studies, including the latest Spitzer Space Telescope surveys (e.g., Enoch et al. 2009; Evans et al. 2009), have found that more than $50 \%$ of the embedded YSOs have $L_{\text {bol }}$ and $\dot{M}_{\text {acc }}$ values considerably lower than those theoretically predicted (i.e. $\sim 2 \times 10^{-6} M_{\odot} \mathrm{yr}^{-1}$ for solarmass YSOs; Shu 1977; Terebey et al. 1984) and roughly of the same order of magnitude as the classical T Tauri stars (CTTs; i.e. $10^{-8} \leq \dot{M}_{\text {acc }} \leq 10^{-6} M_{\odot} \mathrm{yr}^{-1}$; e.g., White \& Hillenbrand 2004). Among several hypotheses, a likely explanation is that the mass accretion is episodic, and the protostars with the lowest luminosities are those observed in quiescent accretion states (Enoch et al. 2009; Evans et al. 2009; Vorobyov 2009). Non-steady mass accretion is often observed in CTTs, such as EXors and FUors (lasting from a few months to several decades), in which $\dot{M}_{\text {acc }}$ increases by several orders of magnitude up to $\dot{M}_{\text {acc }} \sim$ $10^{-4} M_{\odot} \mathrm{yr}^{-1}$ (Hartmann \& Kenyon 1996). It is thus reasonable to believe that a similar mechanism also exists in earlier

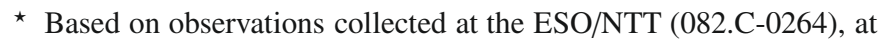
the REM telescope La Silla, Chile, and at the the Italian Telescopio Nazionale Galileo (TNG), operated on the island of La Palma by the Fundacion Galileo Galilei of the INAF (Istituto Nazionale di Astrofisica). and more embedded YSOs. Unfortunately, there is little direct observational evidence of outbursts in Class I YSOs, and so far, only a few clear cases have been detected (e.g. V 1647 Ori outbursts in 2003 and 2008, or OO Ser in 1995; see e.g. Fedele et al. 2007; Kóspál et al. 2007). To reconcile theory with observations and improve the quality of the statistics, it is mandatory to detect and study these rare events.

With this aim, we started a long-term project to monitor the NIR flux and spectroscopic variability of embedded YSOs (mostly Class I and Flat sources) in nearby, young, and active star-forming regions (namely L 1641, CrA, and the Serpens Molecular Cloud). This letter reports the outburst of an embedded YSO in L 1641, namely 2MASS-J05424848-0816347, hereafter [CTF93]216-2 $\left(\alpha_{2000}=05^{\mathrm{h}} 42^{\mathrm{m}} 48.48^{\mathrm{s}}, \delta_{2000}=\right.$ $\left.-08^{\circ} 16^{\prime} 34^{\prime \prime} \cdot 7\right)$. This object was identified by our group as a low-mass embedded YSO (spectral type M, circumstellar $A_{\mathrm{V}} \sim$ $18 \mathrm{mag})$ with a flat spectral index $(\alpha=0.25$, derived by fitting all the photometric data points from 2.2 to $24 \mu \mathrm{m}$ ) and a bolometric luminosity of $\sim 1.9 L_{\odot}$ (Caratti o Garatti et al. in prep., hereafter CoG). It has been named [CTF93]216-2, because it is relatively close to [CTF93]216 (Chen et al. 1993; Chen \& Tokunaga 1994), located about 38.' 5 SW. Our Spitzer/IRAC images indicate that both YSOs have precessing jets, thus they could be part of a wide binary system $(\sim 17300 \mathrm{AU}$, assuming a distance $d=450 \mathrm{pc}$ ). During our recent survey in October 2010 with the robotic telescope REM (see Sect. 2), we detected for [CTF93]216-2 a brightness increase of several magnitudes with respect to the 2 MASS images. We then compared our new 
images with the acquisition image in the $K_{\mathrm{s}}$ band and the NIR spectrum of this source acquired in February 2009, discovering that the outburst was already in progress.

\section{Observations}

NIR spectra were obtained at the ESO-NTT with SofI (Moorwood et al. 1998) (on the 13 Feb. 2009) and at the 3.5-m Italian telescope TNG (13 Oct. 2010) with NICS (Baffa et al. 2001), adopting the usual ABBA configuration. The SofI spectrum was taken with the red grism $(1.51-2.5 \mu \mathrm{m})$, a 0.6 slit $\mathcal{R} \sim 1000)$, and a total integration time of $1800 \mathrm{~s}$. The full width half maximum ( $F W H M$ ) in the dispersion direction, measured from Gaussian fits to the $\mathrm{OH}$ sky lines, was $\sim 19 \AA$ $\left(\sim 260 \mathrm{~km} \mathrm{~s}^{-1}\right)$ in the $K$ band. The NICS low-resolution spectrum was acquired using a slit width of $1^{\prime \prime}(\mathcal{R} \sim 600)$ for the JH and HK grisms (1.15-1.75 $\mu \mathrm{m}$ and $1.4-2.5 \mu \mathrm{m}$, respectively) with a total integration time (Int) of 480 and $160 \mathrm{~s}$ for the $\mathrm{JH}$ and $\mathrm{HK}$ grisms, respectively. The measured $F W H M$ in the dispersion direction was $\sim 33 \AA\left(\sim 600 \mathrm{~km} \mathrm{~s}^{-1}\right)$ in the $K$ band. Telluric and spectrophotometric standards were observed to correct for the atmospheric transmission and flux-calibrate the spectra. The wavelength calibrations were performed using a xenon lamp in the infrared.

Most of our $J, H$, and $K_{\mathrm{s}}$ images were collected between the 10 October and the 6 November 2010 at the $60-\mathrm{cm}$ robotic telescope REM (Zerbi et al. 2001, ESO La Silla observatory) with the NIR camera REMIR (Conconi et al. 2004) and a $150 \mathrm{~s}$ total integration time per filter. An additional $H$ band image was obtained with NICS (13 Oct. 2010) with a 18 s total integration time. Additional $K_{\mathrm{S}}$ photometry (5 Nov. 2003) with the nearIR instrument UIST (Ramsay Howat et al. 2004) was retrieved from the UKIRT data archive $(I n t=15 \mathrm{~s})$. Moreover, another photometric data point was derived from the $K_{\mathrm{s}}$ band of the SofI $K_{\mathrm{s}}$ acquisition image (Int $\left.=6 \mathrm{~s}\right)$. Early epoch photometry was retrieved from the Two-Micron All Sky Survey (2MASS; Skrutskie et al. 2006, J, H, $K_{\mathrm{s}}$ band; November 1998) and from the DEep Near-Infrared southern sky Survey (DENIS; Epchtein et al. 1997, $J$ and $K_{\mathrm{s}}$ bands; January 1997). All the raw data were reduced using IRAF packages, applying standard procedures for sky subtraction, dome flat-fielding, and bad pixel and cosmic ray removal. Photometric calibration was obtained by means of photometric standard stars, except for the SofI $K_{\mathrm{s}}$ acquisition image and the UIST image, where 2MASS photometry of field stars was used.

Finally, we also used additional Spitzer/IRAC photometric data $(3.6,4.5,5.8$, and $8 \mu \mathrm{m}$, obtained on the 8 Oct. 2004), MIPS-24 $\mu \mathrm{m}$ (taken on the 2 Apr. 2005 and 27 Nov. 2008), MIPS 70 and $160 \mu \mathrm{m}$ (2 Apr. 2005), as well as Spitzer/IRS lowresolution spectroscopy $(5.2-39 \mu \mathrm{m}$, obtained on the $12 \mathrm{Mar}$. 2007), presented in CoG. Additional SCUBA/JCMT photometry (at 450 and $850 \mu \mathrm{m}$ ) was taken from Di Francesco et al. (2008).

\section{Results}

\subsection{Photometry}

In Fig. 1, pre- and outburst images of [CTF93]216-2 in the $K_{\mathrm{s}}$ band (top panels) and the Spitzer/MIPS $24 \mu \mathrm{m}$ band (bottom panels) are shown, clearly indicating an increase in the object brightness. Lightcurves in the $J, H, K_{\mathrm{s}}$ bands, and at $24 \mu \mathrm{m}$ are shown in Fig. 2, and the corresponding photometric data points along with their uncertainties are reported in Table 1 . We stress that the photometric data points at $24 \mu \mathrm{m}$ (on the 12 Mar. 2007)

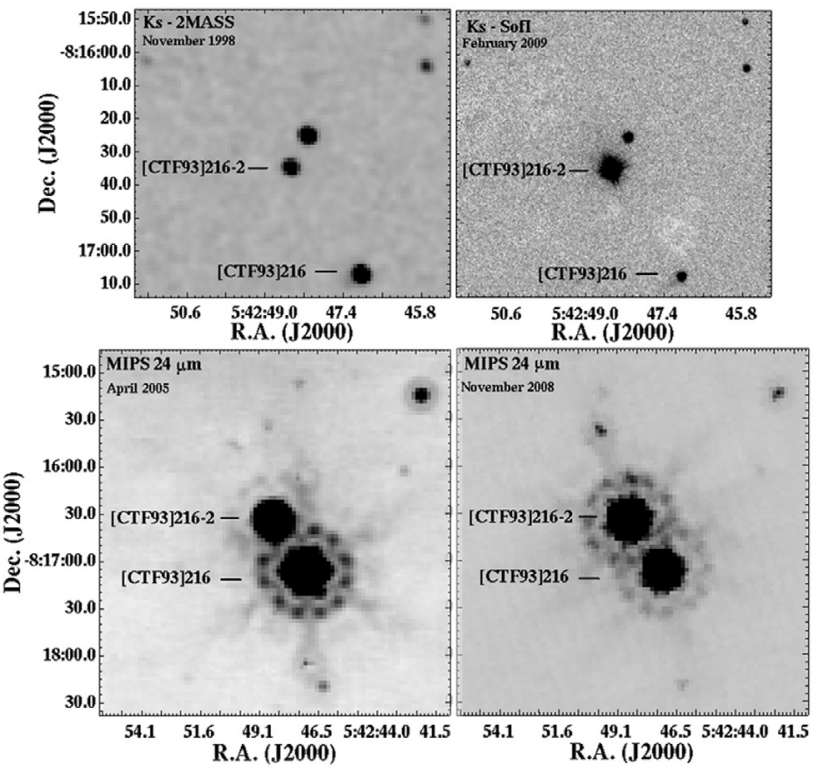

Fig. 1. $K_{\mathrm{s}}$ (top panels) and MIPS-24 $\mu \mathrm{m}$ (bottom panels) images of [CTF93]216-2 and its surroundings before (left) and after (right) the outburst. The position of the YSO [CTF93]216 is also indicated.

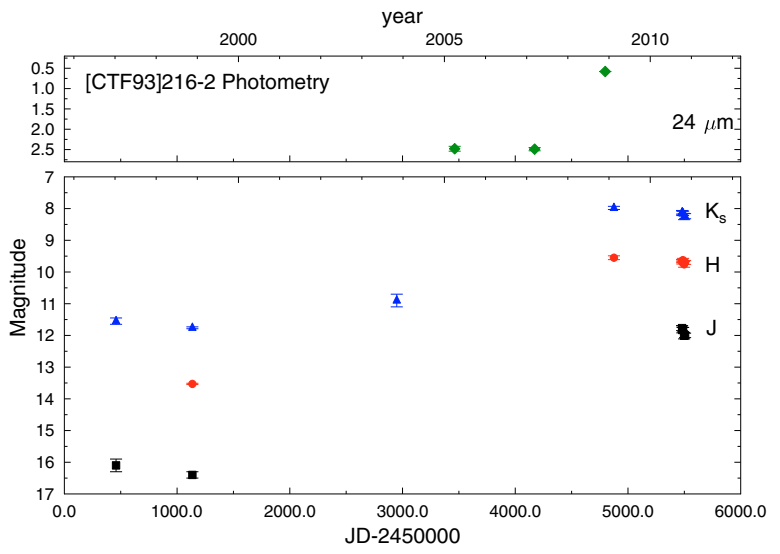

Fig. 2. [CTF93]216-2 lightcurves. $J, H, K_{\mathrm{s}}$, and $24 \mu \mathrm{m}$ data are represented as black squares, red dots, blue triangles, and green diamonds, respectively.

and in the $H$ band (on the 13 Feb. 2009) were derived from the Spitzer/IRS and from the NTT/SofI flux-calibrated spectra, respectively. The $24 \mu \mathrm{m}$ data points (Fig. 2, upper panel) give us some constraints on the date of the outburst, i.e. after March 2007 and before November 2008. Pre-outburst photometry from DENIS, 2MASS, and the UIST $K_{\mathrm{S}}$ images indicates that the object is variable (in both $J$ and $K_{\mathrm{s}}$ bands, we have just one 2MASS data point for the $H$ band), as is often the case for YSOs (Carpenter et al. 2001). Comparing 2MASS (November 1998) with our earliest outburst SofI photometry (February 2009), we measure the amplitudes $\Delta K_{\mathrm{s}} \sim 3.8 \mathrm{mag}$, and $\Delta H \sim 4 \mathrm{mag}$, corresponding to a flux increase $\Delta F$ by a factor of $\sim 33$ and 40, respectively. Considering our earliest postburst photometric point in the $J$ band (October 2010), we derive $\Delta J \sim 4.6 \mathrm{mag}$ (or $\Delta F \sim 69$ ), whereas the $\Delta \mathrm{mag}$ at $24 \mu \mathrm{m}$ is $\sim 1.9$ mag $(\Delta F \sim 6)$. Our data do not allow us to determine the exact date of the peak, but it is clear that the NIR lightcurves are still close to the maximum, and that this plateau phase has lasted 
Table 1. Photometry of [CTF93]216-2.

\begin{tabular}{cccccc}
\hline \hline $\begin{array}{c}\text { Date } \\
\text { (d.m.y) }\end{array}$ & $\begin{array}{c}\text { JD } \\
(2450000+)\end{array}$ & $\begin{array}{c}J \\
(\mathrm{mag})\end{array}$ & $\begin{array}{c}H \\
(\mathrm{mag})\end{array}$ & $\begin{array}{c}\mathrm{Ks} \\
(\mathrm{mag})\end{array}$ & $\begin{array}{c}24 \mu \mathrm{m} \\
(\mathrm{mag})\end{array}$ \\
\hline \hline 10.1 .1997 & 0458.6 & $16.1 \pm 0.2$ & $\ldots$ & $11.55 \pm 0.1$ & $\ldots$ \\
18.11 .1998 & 1135.7 & $16.4 \pm 0.1$ & $13.53 \pm 0.02$ & $11.76 \pm 0.03$ & $\ldots$ \\
5.11 .2003 & 2948.8 & $\ldots$ & $\ldots$ & $10.9 \pm 0.2$ & $\ldots$ \\
2.4 .2005 & 3462.8 & $\ldots$ & $\ldots$ & $\ldots$ & $2.48 \pm 0.1$ \\
12.3 .2007 & 4172.0 & $\ldots$ & $\ldots$ & $\ldots$ & $2.49 \pm 0.06^{a}$ \\
27.11 .2008 & 4798.2 & $\ldots$ & $\ldots$ & $\ldots$ & $0.58 \pm 0.04$ \\
13.2 .2009 & 4875.5 & $\ldots$ & $9.55 \pm 0.06$ & $7.98 \pm 0.05$ & $\ldots$ \\
10.10 .2010 & 5479.9 & $11.77 \pm 0.08$ & $9.66 \pm 0.05$ & $8.13 \pm 0.07$ & $\ldots$ \\
12.10 .2010 & 5481.9 & $11.83 \pm 0.09$ & $9.64 \pm 0.04$ & $8.12 \pm 0.05$ & $\ldots$ \\
13.10 .2010 & 5482.7 & $\ldots$ & $9.70 \pm 0.05$ & $\ldots$ & $\ldots$ \\
19.10 .2010 & 5488.9 & $11.82 \pm 0.07$ & $9.63 \pm 0.06$ & $8.15 \pm 0.07$ & $\ldots$ \\
28.10 .2010 & 5497.8 & $12.01 \pm 0.1$ & $9.76 \pm 0.09$ & $8.25 \pm 0.1$ & $\ldots$ \\
06.11 .2010 & 5506.6 & $12.00 \pm 0.07$ & $9.70 \pm 0.06$ & $8.24 \pm 0.08$ & $\ldots$ \\
\hline
\end{tabular}

Notes. ${ }^{(a)}$ Photometry derived from the Spitzer-IRS flux-calibrated spectrum. ${ }^{(b)}$ Photometry derived from the SofI flux-calibrated spectrum.

at least 2 years, with a small decrement in the $H$ and $K_{\mathrm{s}}$ bands of about 0.1 mag between February 2009 and October 2010. Moreover, our latest photometry (November 2010) seems to indicate that the rate of decrease, between October and November 2010 , has increased to $\sim 0.2 \mathrm{mag} / \mathrm{month}$ in the $J$ band and $\sim 0.1 \mathrm{mag} / \mathrm{month}$ in the $H$ and $K_{\mathrm{s}}$ bands. Pre- and outburst spectral energy distributions (SEDs) are shown in Fig. 3, where all available photometric and spectroscopic data (from this work and from $\mathrm{CoG}$ ) are reported. We note that both the spectral index and bolometric luminosity have changed, as already reported in other YSO outbursts (see e.g. OO Ser, Kóspál et al. 2007). During the outburst, the YSO SED has become bluer and flatter, and the $\alpha$ value, computed between 2.2 and $24 \mu \mathrm{m}$, varied from 0.69 to -0.04 . To compute $L_{\text {bol }}$, and estimate the outburst $\Delta$ mag in the Spitzer/IRAC photometry, we fit the dereddened $\Delta$ mag amplitude from 1.25 to $24 \mu \mathrm{m}$ by means of a power law, obtaining $\Delta m(3.6 \mu \mathrm{m})=2.9 \mathrm{mag}, \Delta m(4.5 \mu \mathrm{m})=2.6 \mathrm{mag}$, $\Delta m(5.8 \mu \mathrm{m})=2.3 \mathrm{mag}$, and $\Delta m(8 \mu \mathrm{m})=2.1 \mathrm{mag}$. We also assume that the SED flux experienced no significant increase for $\lambda>24 \mu \mathrm{m}$. As a result, we estimate an $L_{\text {bol }}$ value of $\sim 22 L_{\odot}$ during the outburst, i.e., $\Delta L_{\text {bol }} \sim 20 L_{\odot}$ with respect to the preoutburst state.

\subsection{Spectroscopy}

Our SofI and NICS low resolution spectra (taken in February 2009 and October 2010, respectively) are shown in Fig. 4 (in black and red).

The SofI spectrum shows an almost featureless continuum, with strong veiling and IR excess. The absorption water band between 1.7 and $2.1 \mu \mathrm{m}$, typical of $\mathrm{M}$ spectral types with a $T_{\text {eff }} \leq 3800 \mathrm{~K}$, is clearly visible. In addition, deep CO bandhead absorption lines are detected at $2.29 \mu \mathrm{m}(2-0), 2.32 \mu \mathrm{m}$ (3-1), and $2.35 \mu \mathrm{m}$ (4-2). Finally, Br $\gamma$ emission, usually associated with accretion, is observed in our SofI spectrum. The measured $\mathrm{Br} \gamma$ flux is $9.6( \pm 1.8) \times 10^{-14} \mathrm{erg} \mathrm{s}^{-1} \mathrm{~cm}^{-2}(E W \sim-3.6 \AA)$, peaking at $2.166 \mu \mathrm{m}$.

The flux of the NICS spectrum, taken 607 days later, shows a slight decrease. The continuum still has a similar shape, but the absorption $\mathrm{H}_{2} \mathrm{O}$ band depth is more prominent. The $E W$ of the CO lines slightly increases by $\sim 1 \AA$ (9.8 to $10.6 \AA$ and 7.2 to $8.2 \AA$, for the $2-0$ and $3-1$ band heads, respectively). More CO lines at $2.38 \mu \mathrm{m}(5-3)$, and $2.41 \mu \mathrm{m}$ (6-4) are detected. In

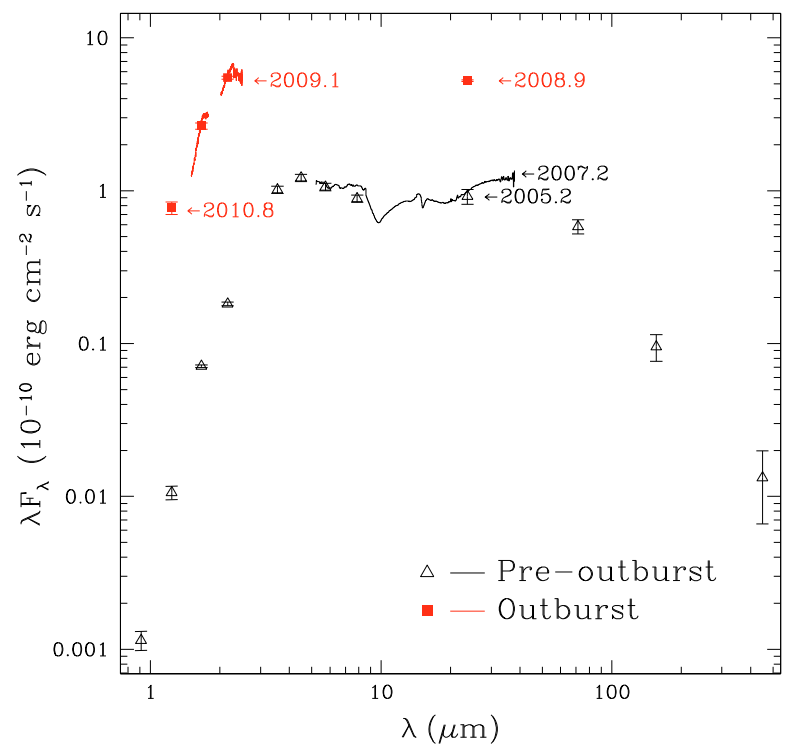

Fig. 3. Spectral energy distribution of [CTF93]216-2. Pre-outburst data: black triangles (photometry), and black line (Spitzer-IRS mid-IR spectrum). Outburst data: red squares (photometry), and red line (SofI NIR spectrum).

addition, a few more narrow-band absorption features become visible on the continuum, i.e. CaI $(2.26 \mu \mathrm{m})$, the $\mathrm{NaI}$ doublet $(2.20-2.21 \mu \mathrm{m}), \mathrm{Mg}$ I $(1.59-1.71 \mu \mathrm{m})$, and the Al I doublet $(1.67-1.68 \mu \mathrm{m})$. No relevant features are detected in the $J$ band, except for the absorption $\mathrm{H}_{2} \mathrm{O}$ band between 1.3 and $1.5 \mu \mathrm{m}$. The $\mathrm{Br} \gamma$ line is not resolved, peaking at $2.166 \mu \mathrm{m}$. The measured flux is $9( \pm 2) \times 10^{-14} \mathrm{erg} \mathrm{s}^{-1} \mathrm{~cm}^{-2}(E W \sim-3.7 \AA)$. We note that no signature of any emission line (such as $\mathrm{H}_{2}$ or [Fe II]) from the jet has been detected on-source in both spectra.

To more tightly constrain the YSO spectral type, we separately compared limited regions of our spectra (in the $H$ and $K$ bands) to model spectra. Veiling and scattering (not taken into account by our fit) are expected to smoothly and slowly vary as a function of wavelength and thus, they should not affect the spectrum in individual bands significantly (e.g., Scholz et al. 2010). For our comparison, we used a series of AMES-DUSTY model spectra (Allard et al. 2001) with $T_{\text {eff }}$ ranging from 2500 to $3900 \mathrm{~K}$ and $\log g=4.0$, as expected for young stars. We varied $T_{\text {eff }}$ to obtain a consistent solution for the two bands, using the $A_{V}=18$ mag derived by CoG. We find a reasonable match between observed and model spectra for $T_{\text {eff }}=3200 \pm 200 \mathrm{~K}$. As an illustration, the model for $3200 \mathrm{~K}$ is shown in Fig. 4 (blue spectrum), shifted by $-0.3 \times 10^{-10} \mathrm{erg} \mathrm{s}^{-1} \mathrm{~cm}^{-2} \mu \mathrm{m}^{-1}$ for clarity.

\section{Discussion}

Our discovery of the [CTF93]216-2 outburst gives us a rare opportunity to study boosted accretion in young embedded protostars, probing whether episodic mass accretion can reconcile the low accretion rates observed in young embedded protostars with the theoretical predictions.

The Bry luminosity, corrected for the circumstellar extinction, is often used to derive an estimate of the accretion luminosity (see e.g. Muzerolle et al. 1998; Natta et al. 2006). Assuming that $A_{\mathrm{V}}=18 \pm 3 \mathrm{mag}$ and $d=450 \mathrm{pc}$, we derive $L(B r \gamma) \sim 3.5(0.9) \times 10^{-3} L_{\odot}$ and, from the Muzerolle et al. (1998) relationship, we obtain $L_{\text {acc }} \sim 22 \pm 8 L_{\odot}$, which is close to the derived $\Delta L_{\mathrm{bol}} \sim 20 L_{\odot}$ and indicates that $L_{\mathrm{acc}} \sim \Delta L_{\mathrm{bol}}$. 


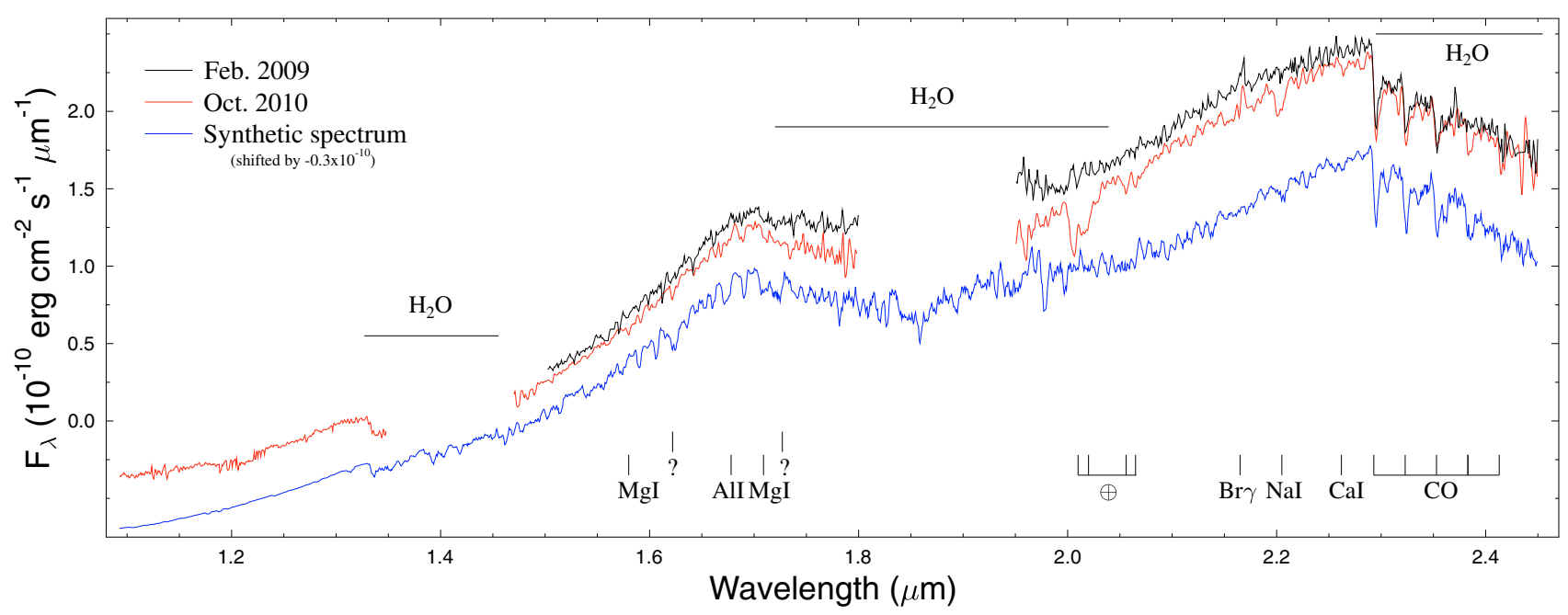

Fig. 4. Flux calibrated NIR spectra of [CTF93]216-2: SofI spectrum (black), and NICS spectrum (red). For comparison, we show an AMESDUSTY model spectrum for effective temperature of $3200 \mathrm{~K}$ and $\log g=4.0$ (Allard et al. 2001). The DUSTY spectrum has been shifted by $-0.3 \times 10^{-10} \mathrm{erg} \mathrm{s}^{-1} \mathrm{~cm}^{-2} \mu \mathrm{m}^{-1}$ for clarity. The detected features are also labelled.

On the other hand, $\dot{M}_{\text {acc }}$ can be derived from $\dot{M}_{\text {acc }}=L_{\text {acc }} \times$ $1.25 R_{*} / G M_{*}$ (Gullbring et al. 1998), where $M_{*}$ and $R_{*}$ are the stellar mass and radius, and the number 1.25 is derived by assuming a value of $5 R_{*}$ for the inner radius of the accretion disk.

Assuming that $T_{\mathrm{eff}}=3200 \pm 200 \mathrm{~K}$ and $L_{*} \sim 1.9 \pm 0.1 L_{\odot}$, from Siess et al. (2000) models, we obtain $M_{*}=0.24 \pm 0.04 M_{\odot}$ and $R_{*}=4.4 \pm 0.4 R_{\odot}$, which gives a mass accretion rate of $\sim 1.1-1.3 \times 10^{-6} M_{\odot} \mathrm{yr}^{-1}$. While $M_{*}$ is well constrained by $T_{\text {eff }}$, the radius $R_{*}$ depends on $L_{*}$. Thus this last should be considered as an upper limit, since pre-outburst $L_{\text {acc }}$ is unknown. However, even a $50 \%$ decrease in $L_{*}$ would affect $R_{*}$ and thus $\dot{M}_{\text {acc }}$ of just $20 \%$.

Compared to typical accretion rates of Class I YSOs with similar masses (i.e. $10^{-8} M_{\odot} \mathrm{yr}^{-1}$; e.g., CoG; Scholz et al. 2010; White \& Hillenbrand 2004), the derived $\dot{M}_{\text {acc }}$ is about two orders of magnitude higher. This value is about an order of magnitude lower than what some episodic-accretion models predict for these early YSO bursts (i.e. $10^{-5} \mathrm{M}_{\odot} \mathrm{yr}^{-1}$; e.g. Vorobyov 2009), which should resemble the more evolved FUor counterparts. On the other hand, we note that the magnitude of luminosity change during this outburst $(\sim 10)$ is similar to those of V 1647 Ori and OO Ser $(\sim 8)$. Our object would probably differ from the others in its pre-outburst $L_{\text {bol }}$, which is about two times lower, because of its lower mass. Moreover, as already noted in previous cases (e,g. Hodapp et al. 1996; Fedele et al. 2007; Kóspál et al. 2007), the [CTF93]216-2 outburst shows similarities with both FUor and EXor events, i.e. a NIR featureless FUor spectrum with strong absorption $\mathrm{CO}$ band heads, but with $\mathrm{Br} \gamma$ line in emission as in EXors. The event duration (if confirmed by additional observations) and amplitude are in-between those of EXors and FUors, thus, as noted by other authors (Gibb et al. 2006; Fedele et al. 2007) EXors and FUors might not be distinct categories of eruptive events, but instead part of a continuum of outburst events.
Acknowledgements. A.C.G. was supported by the Science Foundation of Ireland, grant 07/RFP/PHYF790. We would like to thank Dino Fugazza and Francesca Ghinassi for supporting the REM and TNG observations.

\section{References}

Allard, F., Hauschildt, P. H., Alexander, D. R., Tamanai, A., \& Schweitzer, A. 2001, ApJ, 556, 357

Andre, P., Ward-Thompson, D., \& Barsony, M. 1993, ApJ, 406, 122

Baffa, C., Comoretto, G., Gennari, S., et al. 2001, A\&A, 378, 722

Carpenter, J. M., Hillenbrand, L. A., \& Skrutskie, M. F. 2001, AJ, 121, 3160

Chen, H., \& Tokunaga, A. T. 1994, ApJS, 90, 149

Chen, H., Tokunaga, A. T., \& Fukui, Y. 1993, ApJ, 416, 235

Conconi, P., Cunniffe, R., D'Alessio, F., et al. 2004, in SPIE Conf. Ser., ed. A. F. M. Moorwood, \& M. Iye, 5492, 1602

Di Francesco, J., Johnstone, D., Kirk, H., MacKenzie, T., \& Ledwosinska, E. 2008, ApJS, 175, 277

Enoch, M. L., Evans, N. J., Sargent, A. I., \& Glenn, J. 2009, ApJ, 692, 973

Epchtein, N., de Batz, B., Capoani, L., et al. 1997, The Messenger, 87, 27

Evans, N. J., Dunham, M. M., Jørgensen, J. K., et al. 2009, ApJS, 181, 321

Fedele, D., van den Ancker, M. E., Petr-Gotzens, M. G., \& Rafanelli, P. 2007, A\&A, 472, 207

Gibb, E. L., Rettig, T. W., Brittain, S. D., et al. 2006, ApJ, 641, 383

Gullbring, E., Hartmann, L., Briceno, C., \& Calvet, N. 1998, ApJ, 492, 323

Hartmann, L., \& Kenyon, S. J. 1996, ARA\&A, 34, 207

Hodapp, K., Hora, J. L., Rayner, J. T., Pickles, A. J., \& Ladd, E. F. 1996, ApJ, 468,861

Kóspál, Á., Ábrahám, P., Prusti, T., et al. 2007, A\&A, 470, 211

Lada, C. J., \& Wilking, B. A. 1984, ApJ, 287, 610

Moorwood, A., Cuby, J.-G., \& Lidman, C. 1998, The Messenger, 91, 9

Muzerolle, J., Hartmann, L., \& Calvet, N. 1998, AJ, 116, 2965

Natta, A., Testi, L., \& Randich, S. 2006, A\&A, 452, 245

Ramsay Howat, S. K., Todd, S., Leggett, S., et al. 2004, in SPIE Conf. Ser., ed. A. F. M. Moorwood, \& M. Iye, SPIE Conf. Ser., 5492, 1160

Scholz, A., Wood, K., Wilner, D., et al. 2010, MNRAS, 1258

Shu, F. H. 1977, ApJ, 214, 488

Siess, L., Dufour, E., \& Forestini, M. 2000, A\&A, 358, 593

Skrutskie, M. F., Cutri, R. M., Stiening, R., et al. 2006, AJ, 131, 1163

Terebey, S., Shu, F. H., \& Cassen, P. 1984, ApJ, 286, 529

Vorobyov, E. I. 2009, ApJ, 704, 715

White, R. J. \& Hillenbrand, L. A. 2004, ApJ, 616, 998

Zerbi, R. M., Chincarini, G., Ghisellini, G., et al. 2001, Astron. Nachr., 322, 275 